\title{
SGTD: Sistema de Gerenciamento de Tráfego Distribuído para Redes Veiculares
}

\author{
Thiago S. Gomides, Massilon L. Fernandes, Pedro H. Souza e Daniel L. Guidoni \\ ${ }^{1}$ Departamento de Ciência da Computação \\ Universidade Federal de São João Del Rei (UFSJ), MG - Brasil
}

\{gomides, massilon, pedronhds, guidoni\}@ufsj.edu.br

\begin{abstract}
The mobility management is an important and recurrent challenge in the urban centers, mainly, because intensification of population grouping in the big cities. In this sense, one of the reflexes about the question be in the disproportionate growth of the vehicle number in relation to urban infrastructure, responsible for emergence or intensification of traffic jams. The objective of this work is proposed a fully distributed algorithm capable of reducing these impacts from the of vehicular reorganization flow. Therefore, the vehicles, in the collaborative form, are responsible by classification and information sharing about the displacements carried out for decision-making in a distributed environment. Simulation results indicate that solution shows is able reduce the travel time, the congestion time, besides increasing the average speed with a small impact on the number of transmitted message, in order to enable a good performance of proposed system.
\end{abstract}

Resumo. O gerenciamento da mobilidade é um importante e recorrente desafio dos centros urbanos, principalmente, devido à intensificação do agrupamento populacional nas grandes cidades. Nesse sentido, um dos reflexos desse impasse está no crescimento desproporcional do número de veículos em relação à infraestrutura urbana, responsável pelo surgimento ou pela intensificação dos congestionamentos. O objetivo deste trabalho é propor um algoritmo totalmente distribuído capaz de reduzir esses impactos a partir da reorganização do fluxo veicular. Dessa forma, os veículos, de maneira colaborativa, são responsáveis pela classificação e pelo compartilhamento de informações sobre os deslocamentos realizados para a tomada de decisão em um ambiente distribuído. Os resultados das simulações indicam que a solução apresentada consegue reduzir o tempo de viagem, o tempo em congestionamentos, além de aumentar a velocidade média alcançada com baixo impacto no número de mensagens transmitidas, de modo a possibilitar um bom desempenho do sistema proposto.

\section{Introdução}

Aprimorar a mobilidade urbana de pessoas e cargas é um desafio enfrentado por vários países, em que a Organização das Nações Unidas (ONU), por meio do projeto $\mathrm{ONU} \mathrm{Ha-}$ bitat [ONU-HABITAT 2018], promove frequentes discussões entre os governos, pesquisadores e ambientalistas com foco na resolução desse problema. A utilização preferencial da população pelos transportes individuais em relação aos coletivos contribui para 
a intensificação dessa situação. Nesse sentido, inúmeros são os reflexos dessa questão e, no âmbito econômico, acredita-se que nos EUA o custo de congestionamentos ultrapassará 192 bilhões de dólares até 2020 [Scorecard 2015]. Além disso, segundo estudos da Organização Mundial da Saúde (OMS), a poluição advinda da queima dos combustíveis fósseis propicia o surgimento de doenças, sendo estas: o aumento da ocorrência de câncer de pulmão, das doenças cardíacas, do número de acidentes vasculares cerebrais e a intensificação das enfermidades respiratórias [Organization et al. 2006].

Dessa forma, busca-se a a redução de congestionamentos a partir do desenvolvimento de técnicas capazes de reorganizar o fluxo dos veículos com baixo impacto econômico e ambiental. Nesse contexto, mudanças sociais são propostas em função do menor custo para implantação, no entanto, alternativas como o rodízio de placas e o incentivo ao uso de transportes públicos são pouco eficientes [Cunha et al. 2017]. Nesse contexto, populariza-se nos grandes centros urbanos a utilização de sistemas de navegação veicular (VNS - Vehicle Navigation System) que, por princípio, recomendam a rota mais rápida sem avaliação prévia do contexto do deslocamento. Desse modo, os VNSs como WAZE, Google Maps, INRIX, ou Apple Maps se limitam à análise de um conjunto de características na reorganização do fluxo dos veículos, sobretudo, não avaliam a dimensão das vias secundárias, o que potencializa o surgimento ou deslocamento dos congestionamentos existentes [Thai et al. 2016]. Além disso, esses serviços dependem de informações coletadas em redes sociais, que podem não representar o real estado das condições de tráfego das vias [de Souza et al. 2017a].

Com o avanço da comunicação móvel, especialmente das tecnologias de comunicação sem fio e processamento embarcado nos veículos, é possível o desenvolvimento de um sistema de transporte inteligente (ITS) com o objetivo de utilizar a comunicações entre veículos para resolver diversos problemas nos transportes tradicionais dos centros urbanos como, por exemplo, reduzir engarrafamentos, diminuir o tempo de viagem dos veículos, diminuir acidentes, aumentar a capacidade de fluxo das vias etc [Lana et al. 2018]. Para isso, as Redes Veiculares Ad-Hoc (VANETs - Vehicular Ad-Hoc NEtworks) compõem um conjunto de serviços de comunicação onde uma rede ad-hoc sem fio é criada entre veículos [Ahmed and Gharavi 2018, Wu et al. 2015, Dietzel et al. 2014]. Um serviço de gerenciamento de tráfego distribuído pode ser criado sob a infraestrutura física de comunicação dos veículos, onde cada veículo monitora a sua percepção da condição do tráfego da sua via (velocidade média do veículo, quantidade de carros dentro do seu raio de comunicação etc) e dissemina esse conjunto de informação para os demais veículos [Pisa et al. 2018, de Souza et al. 2017a]. O objetivo da disseminação da informação é a criação de um conhecimento distribuído sobre as condições de tráfego das vias de uma determinada região. Dessa forma, veículos podem encontrar rotas alternativas para reduzir congestionamentos e, por conseguinte, reduzir o tempo de viagem. O principal desafio do gerenciamento de tráfego é modificar a rotas dos veículos sem causar congestionamentos em outras regiões da cidade [de Souza et al. 2017a].

O objetivo deste trabalho é propor o SGTD, um Sistema de Gerenciamento de Tráfego totalmente Distribuído capaz de classificar e reorganizar o fluxo de veículos para reduzir congestionamentos. A solução proposta fundamenta-se na distância percorrida durante o tempo de percurso em relação àquela esperada quando os veículos estão 
em deslocamento considerando as velocidades máximas das vias. Com o objetivo de criar um conhecimento distribuído sobre as condições de tráfego, foi proposto um mecanismo de compartilhamento de informações, onde informações sobre as condições das vias são compartilhadas com base na alteração de tráfego percebida pelo veículo em conjunto com as informações já disseminadas por outros veículos na mesma região. $\mathrm{O}$ mecanismo de compartilhamento proposto também possui como objetivo a redução da quantidade de mensagens para a criação do conhecimento distribuído, onde o compartilhamento é limitado para a vizinhança do veículo. Utilizando o conhecimento adquirido, o veículo verifica se exite rotas com um menor tempo de viagem. A solução proposta foi comparada com o algoritmo PANDORA [de Souza et al. 2017b], que é uma solução distribuída para o gerenciamento de tráfego. Além disso, foi realizada a comparação com o (RkSP) [Pan et al. 2017], que possui uma visão centralizada das condições de tráfego e FOX [Brennand et al. 2017], que utiliza o conceito de computação em névoa. Os algoritmos foram avaliados em termos de mensagens transmitidas e características de tráfego, onde o SGTD é capaz de encontrar resultados superiores em comparação com os algoritmos da literatura.

As seções do artigo estão organizadas da seguinte forma. Os principais trabalhos relacionados são apresentados na seção 2. A solução proposta é apresentada na seção 3 . A comparação dos algoritmos é apresentada na seção 4 e a conclusão do trabalho é apresentada na seção 5 .

\section{Trabalhos Relacionados}

O desenvolvimento de soluções em VANETs para gerenciamento de tráfego tem ganhado muita atenção nos últimos anos. Em geral, essas aplicações possuem como objetivo reduzir os congestionamentos com baixo impacto na infraestrutura urbana a partir da utilização de serviços e tecnologias de comunicação entre os veículos [Ahmed and Gharavi 2018]. A redução do congestionamento é encontrada por meio da alteração das rotas dos veículos. Nesse sentido, classifica-se as soluções em relação ao modelo de comunicação e organização dos automóveis, que podem ser Centralizadas, Descentralizadas ou Distribuídas. Propostas semelhantes à apresentada neste artigo são encontradas na literatura em: Centralizadas - DSP e RkSP [Pan et al. 2017], Descentralizadas FOX [Brennand et al. 2017] e Distribuída - PANDORA [de Souza et al. 2017b], apresentadas abaixo.

Em [Pan et al. 2017], os autores apresentam as soluções DSP (Dynamic Shortest Path) e R $k$ SP (Random $k$ Shortest Path) como um conjunto de serviços centralizados, fundamentados na duração do deslocamento nas vias para o cálculo de novas rotas. Nesse sentido, com o objetivo de proporcionar conhecimento suficiente para a reorganização do fluxo veicular, nas duas propostas, cada veículo (ao passar por interseções entre as ruas) notifica o servidor central o tempo de viagem verificado durante o seu percurso. No DSP, em intervalos periódicos, o servidor encaminha novas rotas de menor custo aos veículos calculadas a partir das informações coletadas. Entretanto, devido ao processamento centralizado e global da rede, a reorganização é realizada individualmente sem qualquer mecanismo para o balanceamento de carga, o que potencializa o deslocamento dos congestionamentos existentes. Nesse sentido, para uma distribuição mais uniforme do fluxo veicular, a solução R $k$ SP complementa o mecanismo de cálculo de novas rotas por meio da criação de $k$ caminhos alternativos, ainda que esta decisão não apresente o tra- 
jeto de menor custo. Assim, como exposto em [Pan et al. 2017], as soluções conseguem reduzir os tempo de congestionamentos e o algoritmo $\mathrm{R} k \mathrm{SP}$ possui melhores resultados, o que também foi observado em nossas avaliações.

O Fast Offset Xpath (FOX) é apresentado em [Brennand et al. 2017] como um protocolo de roteamento descentralizado com processamento e armazenamento de conhecimento a partir de estruturas denominadas névoas. Nesse sentido, o modelo de computação em névoa utilizado realiza a distribuição geográfica de equipamentos com capacidade de comunicação sem fio denominados de Road-Side Unit - RSU. A deposição das RSUs considera a divisão da cidade em regiões de mesmo tamanho, proporcionando total cobertura de comunicação ao posicionar centralmente as RSUs em cada região. No FOX, os veículos durante seus trajetos notificam a RSU correspondente a sua região sobre as condições de trânsito observadas, assim como solicitam periodicamente rotas alternativas com menores tempos de viagens. Dessa forma, cada RSU, provida de informações a respeito de sua área de conhecimento, analisa a existência de rotas menos congestionadas por meio do algoritmo de $k$ rotas alternativas e, posteriormente, notifica os veículos com as melhores opções. Segundo os autores, a descentralização do processamento e armazenamento proporciona uma proximidade entre o serviço e o usuário, reduzindo o tempo de resposta e a complexidade da manutenção das informações sobre as vias.

Em [de Souza et al. 2017b] é apresentado o PANDORA, um sistema de gerenciamento de tráfego avançado, totalmente distribuído e que realiza o compartilhamento de conteúdo de forma oportunista. Nesse trabalho, a reorganização dos veículos é realizada em regiões denominadas áreas críticas, em que torna-se necessário o conhecimento das características do fluxo de veículos no cenário. Ao receber o conhecimento das áreas críticas, os veículos realizam o roteamento de forma individual e periódica. Para isso, dois mecanismos de disseminação de informações são aplicados, sendo esses: o compartilhamento de mensagens de sinalização (Beacon) e as transmissões de conhecimento direcionadas (Unicast) nas áreas críticas. O PANDORA foi avaliado em uma região de $5 \mathrm{~km}^{2}$ contendo uma região cŕitica de $1 \mathrm{~km}^{2}$, apresentando melhores resultados em comparação com soluções da literatura.

\section{SGTD}

O SGTD é um protocolo de roteamento de veículos fundamentado na construção do conhecimento distribuído a partir das percepções locais do fluxo e das características das vias e, consequentemente, elimina a necessidade das infraestruturas auxiliares de comunicação. A figura 1 apresenta o funcionamento geral da solução proposta, que é composta por três módulos distintos. No módulo Classificação do Congestionamento, o veículo calcula o nível de retenção em relação às distâncias percorridas na via e àquelas esperadas em fluxo livre. Em seguida, estes encaminham os níveis de retenção detectados aos nós vizinhos em busca da criação de uma base de dados distribuída utilizando o módulo Compartilhamento das Estimativas. Por fim, no módulo Decisão de Novas Rotas os veículos, ao analisarem as informações recebidas, definem novas rotas considerando os menores níveis de retenção.

As próximas seções discutem os três módulos descritos. Para o seu correto funcionamento, cada veículo dispõem de informações de geolocalização, do mapa digital da cidade e das características específicas de cada via, como dimensões e velocidade máxima permitida. 


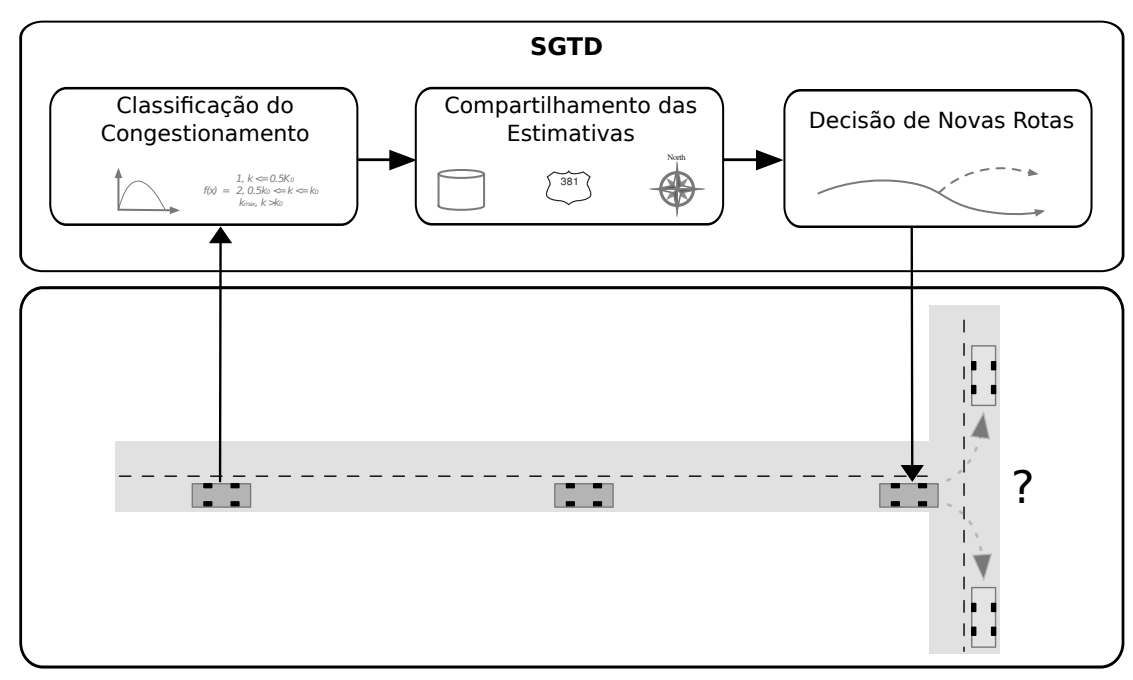

Figura 1. Funcionamento básico do SGTD

\subsection{Classificação de Congestionamento}

O processo de categorizar os ambientes congestionados consiste na utilização de técnicas capazes de mensurar o fluxo veicular com o objetivo de prover mecanismos que auxiliem nas decisões de realocação do trânsito e, assim, minimizar os possíveis efeitos dos congestionamentos. Dessa forma, a classificação proposta neste trabalho é realizada a partir da definição de níveis de retenção, onde cada veículo, ao analisar as condições do tráfego da via, torna-se capaz de verificar o grau correspondente à percepção do seu deslocamento. Nesse sentido, a medição da retenção do fluxo foi divida em dois segmentos: Análise do Deslocamento (3.1.1) e Classificação do Congestionamento (3.1.2). No estudo do primeiro, cada automóvel avalia as percepções momentâneas a respeito do fluxo, enquanto, no segundo, há a preocupação em quantizar o trânsito quanto ao histórico do trajeto do veículo na via.

\subsubsection{Análise do Deslocamento}

Em ambientes de fluxo veicular intenso, normalmente o deslocamento ocorre em velocidades reduzidas e são percebidas constantes interrupções no trânsito, o que implica diretamente na redução da distância percorrida pelos automóveis. Nesse sentido, para mensurar as retenções do fluxo, foi constatado que os ambientes congestionados possuem menores expectativas de deslocamento, ou seja, a extensão do trajeto dos veículos é frequentemente menor considerando um intervalo de tempo. Dessa forma, a análise do deslocamento proposta é realizada por meio da associação entre a distância esperada e aquela verificada durante o percurso, chamada de distância percorrida. Para realizar essa avaliação foram definidos parâmetros e coeficientes apresentados a seguir:

- Tempo de Percurso $\left(t_{p}\right)$ : o tempo percorrido por um veículo ao locomover-se em uma via.

- Distância Esperada (DE): a distância prevista (em condições de fluxo livre) considerando $t_{p}$ e a velocidade máxima da via.

- Distância Percorrida (DP): a dimensão do percurso realizada durante o $t_{p}$. 
- Fator de Contenção (FC): define a percepção momentânea da expectativa do deslocamento.

A figura 2 apresenta o estudo do deslocamento de um veículo ao percorrer a via AB. Dado o tempo de percurso $\left(t_{p}\right)$, espera-se, em condições de fluxo livre (percurso em velocidade máxima), que o veículo tenha percorrido a distância DE. No entanto, em vista das retenções no fluxo ocasionadas por automóveis que se deslocam em velocidades inferiores às máximas permitidas ou devido às pequenas alterações do deslocamento, sem que haja a ocorrência de congestionamentos, propusemos o intervalo $\triangle \mathbf{D E}$ (faixa verde na figura 2). Este intervalo, tem como objetivo reduzir a interferência das pequenas variações e classificar o distanciamento limite (em relação à DE) para que o trânsito seja identificado como fluxo livre. Portanto, $\triangle \mathbf{D E}$ define uma flutuação esperada em torno da distância percorrida em fluxo livre, minimizando a incidência de classificações de erradas.
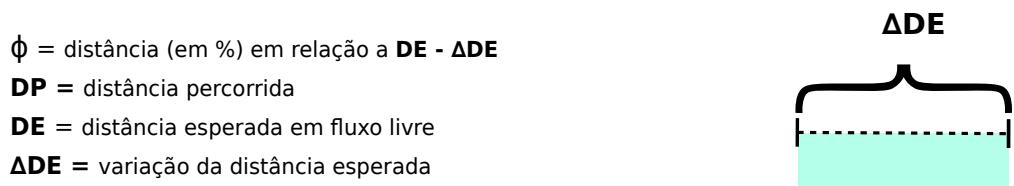

DE

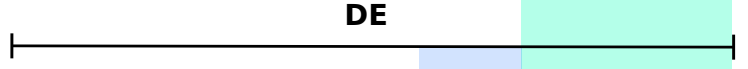

A

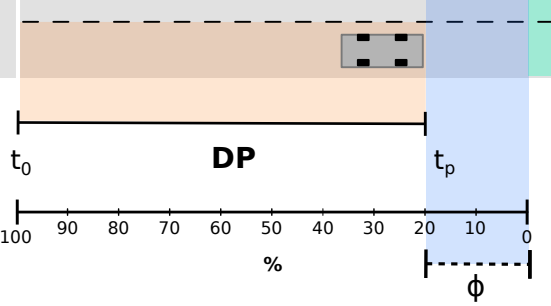

Figura 2. Análise do Deslocamento

Dessa forma, veículos com uma distância percorrida DP contida no intervalo $\triangle$ DE são observados como deslocamento em fluxo livre. Por outro lado, veículos com deslocamentos inferiores ao observado em fluxo livre são caracterizado por um fator de contenção (FC). Este fator é calculado considerando, em porcentagem, a distância que o veículo se encontra em relação ao limite inferior da faixa de fluxo livre (faixa azul na figura 2) e definida pelo valor $\phi$. Na Figura 2, por exemplo, a distância percorrida está $20 \%$ inferior àquela esperada, o que implica na elevação do fator de contenção. Dessa forma, a expansão dos congestionamentos está relacionada ao aumento da proporção entre as distâncias (valor de $\phi$ ) e o valor do $F C$ define a medição momentânea da contenção observada, apresentado a seguir.

\subsubsection{Classificação do Congestionamento}

Durante o deslocamento, os veículos classificam suas percepções momentâneas do fator de contenção a cada unidade de tempo e conforme a função de crescimento ilustrado na figura 3(a). O eixo-x da figura representa a variação do valor de $\phi$ e o eixo-y representa um 


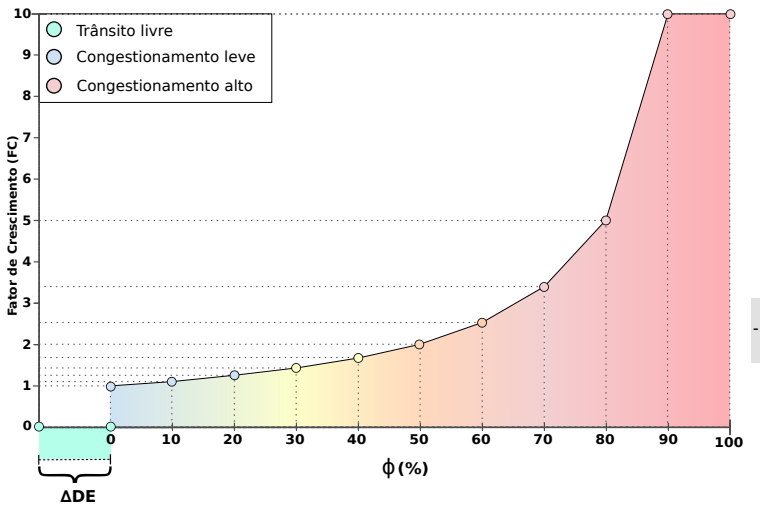

(a) Fator de contenção Momentâneo

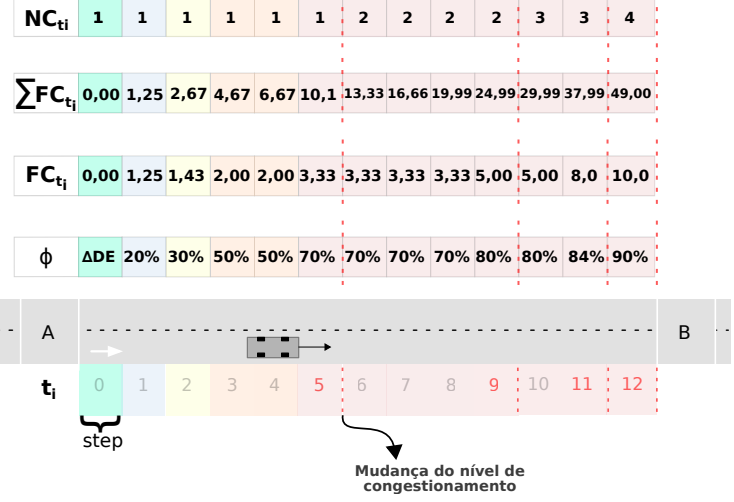

(b) Classificação do Congestionamento

Figura 3. Classificação dos Congestionamentos

fator de contenção (FC) que varia de 0 a 10. Foi definido que, para pequenos e moderados valores de $\phi(0-50 \%)$, o fator de contenção possui um crescimento lento (0 a 2). Para $\phi>50 \%$, o fator de contenção cresce rápido (2 a 10), uma vez que o congestionamento está aumentando rapidamente.

A classificação momentânea da contenção do trânsito é utilizada para definir o Nível de Congestionamento (NC) da via, onde NNC define o Número de Níveis de Congestionamento. $\mathrm{NC}$ é uma variável positiva e inteira, onde $\mathrm{NC}=1$ define que uma via possui trânsito livre e $\mathrm{NC}=\mathrm{NNC}$ define que a via possui um congestionamento alto. Os níveis de congestionamentos definem uma quantificação confiável do trânsito, onde consideram-se um conjunto de medições momentâneas da contenção do trânsito em seu cálculo. Os NCs são utilizados nos cálculos de pesos das vias com o objetivo de se encontrar novas rotas. Sempre que o veículo inicia o percurso em uma nova via e a cada intervalo de tempo $t_{i}$, ele calcula o Nível de Congestionamento $\left(\mathrm{NC}_{v i a}\right)$ com base na fórmula 1, onde $F C_{t}$ representa o fator de contenção no tempo $t$ e $\mathrm{NNC}$ representa o Número de Níveis de Congestionamentos.

$$
N C_{v i a}=\stackrel{\substack{\text { fluxo livre } \\ 1}}{\Gamma}\left\lfloor\frac{1}{\mathrm{NCC}} \cdot \sum_{i=0}^{t_{\max }} F C_{t_{i}}\right\rfloor
$$

Se todos os fatores de contenção $\left(F T_{t_{i}}\right)$ considerados pelo veículo são 0 , o veículo está percorrendo a via em fluxo livre e o $N C_{v i a}=1$. Caso contrário, $\frac{\sum F C_{t_{i}}}{N N C}$ será diferente de zero e um acréscimo no nível é realizado. Entretanto, apenas a parte inteira do acréscimo é considerada. Isso é feito para reduzir interferências de pequenas variações no fator de contenção. Se $\frac{\sum F C_{t_{i}}}{N N C}>N C C$, então $N C_{v i a}=N C C$, uma vez que NCC define o nível máximo de congestionamento. Quando o veículo verifica que houve mudança no nível de congestionamento da via, uma mensagem é enviada para notificar os veículos vizinhos. A Figura 3(b) apresenta a variação $\phi$ entre as distancias, a classificação momentânea do Fator de Contenção $\left(F C_{t_{i}}\right)$, a soma dos fatores de contenção $\left(\sum F C_{t_{i}}\right.$ ), o nível de congestionamento verificado pelo veículo e as detecções das mudanças dos níveis de congestionamento (linhas verticais pontilhadas em vermelho). A cada intervalo de tempo (denominado por $t_{i}=0,1,2,3, \ldots, t_{\max }$ ), o veículo 
realiza as medições e, após $t_{i}=5$, temos $\sum F C_{t_{i}}=10,01 \mathrm{e}$, utilizando a equação 1 , temos $N C_{\text {via }}=1+\left\lfloor\frac{1}{10} \cdot 10,01\right\rfloor \longrightarrow N C_{\text {via }}=1+\lfloor 1,001\rfloor \longrightarrow N C_{\text {via }}=2$. Considerando os momentos $t_{i}=6,7$ e 8 , os valores de $\sum F C_{t_{i}}$ avaliados na equação 1 definem um $N C_{v i a}=2$. Entretanto, quando $t_{i}=9$ o veículo verifica $\sum F C_{t_{i}}=24,99$, onde temos $N C_{\text {via }}=1+\left\lfloor\frac{1}{10} \cdot 24,99\right\rfloor \longrightarrow N C_{\text {via }}=1+\lfloor 2,49\rfloor \longrightarrow N C_{\text {via }}=3$. Outras mudanças de níveis ocorrem quanto $t_{i}=11$ e 12 .

\subsection{Compartilhamento de Informação}

Sempre que um veículo verifica uma mudança do nível de congestionamento, é necessário realizar o compartilhamento dessa informação para os veículos vizinhos de modo a obter um conhecimento distribuídos sobre as condições das vias. Esse compartilhamento é feito utilizando mensagens do tipo beacons contendo uma tupla $\left\langle\right.$ via, $N C_{v i a}>$, onde via representa o ID da via que o veículo se encontra, $N C_{\text {via }}$ representa o nível de congestionamento observado pelo veículo na via considerada. A mensagem beacons possui $\mathrm{TTL}=1$, ou seja, ela não é retransmitida pelos vizinhos.

Os veículos dispõem de uma tabela com as estimativas recebidas e observadas durante o percurso e as atualizações são efetuadas a partir da existência de dados mais recentes. Com objetivo de encaminhar eficientemente as estimativas sem que exista sobrecarga de mensagens ou carência de conhecimento, ou seja, supressão do compartilhamento de informação, foram propostos os módulos (3.2.1) Disseminação do Conhecimento e (3.2.2) Manutenção do Conhecimento, apresentados a seguir.

\subsubsection{Disseminação do Conhecimento}

A metodologia proposta para conter a sobrecarga de mensagens é iniciada quando os veículos verificam a mudança de via e, posteriormente, identificam a existência de alterações no nível de congestionamento. Detectadas as modificações, os veículos consultam suas respectivas bases de dados para verificar a existência ou não do nível de congestionamento da via em questão. Quando a informação não é encontrada, a estimativa calculada deve ser armazenada e enviada para os vizinhos pois, a princípio, nenhum outro veículo detectou e compartilhou o congestionamento verificado.

Se o veículo detectar a existência de uma análise prévia da via em questão em sua base de dados (seja pelo recebimento da informação compartilhada por outro veículo ou por uma atualização anterior), o veículo realiza a comparação entre os níveis de congestionamentos. Se os níveis forem iguais, a disseminação da informação é contida. Por fim, sempre que o conhecimento armazenado em sua base de dados for diferente em relação ao nível calculado, a disseminação da informação é realiza, pois acredita-se que o fluxo de veículos esteja em modificação e tais estimativas reflitam o cenário mais atual da via.

\subsubsection{Manutenção do Conhecimento}

A Disseminação do Conhecimento também possui como objetivo minimizar o número de mensagens para criar um conhecimento distribuído sobre as condições do tráfego da via. Entretanto, deve existir um equilíbrio entre esse controle e a perpetuação da informação 
sobre a via, uma vez que os veículos continuam o seu percurso após passar por uma determinada via, porém, novos veículos irão passar pela via e necessitarão de informações. Para isso, um mecanismo de manutenção do conhecimento foi criado com o objetivo de prevenir que as estimativas a respeito do Nível de Congestionamento em qualquer via sejam extintas.

Para isso, a cada $\mathbf{T}$ unidades de tempo verificados em uma determinada via, o veículo realiza o compartilhamento do nível de congestionamento verificado até o momento, ou seja, o nível de congestionamento da via é compartilhado pelo veículo sem que haja uma alteração de nível. Porém, para minimizar a duplicação de mensagens, sempre que novas informações referentes a via em que o veículo se desloca são recebidas, é realizado o cancelamento do compartilhamento, pois acredita-se que a via atual tem conhecimento suficiente e não demanda a sua manutenção. É importante ressaltar que a manutenção de conhecimento é realizada somente quando não há alteração nos níveis de congestionamentos de uma via no intervalo de $\mathbf{T}$ unidades de tempo.

\subsection{Decisão de Novas Rotas}

Na solução proposta, cada veículo realiza o cálculo distribuído de uma nova rota ao se aproximar das interseções. Isso é feito para que o veículo possa receber a maior quantidade de informação sobre vias vizinhas para verificar se existem rotas alternativas. Em outras palavras, a decisão tardia pode contribuir para a utilização de níveis de conhecimentos mais atualizados. Para verificar a existência de uma nova rota, cada veículo modela o mapa da cidade como um grafo dirigido e ponderado, onde as arestas representam as vias e os vértices representam interseções entre duas ou mais vias. $\mathrm{O}$ ponderamento das arestas é feito segundo a equação 2 , onde peso $_{i j}$ representa o peso da via (aresta) $i j$, ou seja, a aresta entre os vértices $i$ e $j, D_{i j}$ representa o tamanho da via $i j, V_{\text {max }_{i j}}$ representa a velocidade máxima da via $i j$ e $N C_{i j}$ representa o nível de congestionamento observado da via em questão. Nesse sentido, busca-se manter a proporcionalidade entre o nível de congestionamento, a dimensão do segmento $\left(D_{i j}\right)$ e a velocidade máxima permitida na via $\left(V_{\max _{i j}}\right)$. Assim, o nível de congestionamento da via é um fator multiplicador em seu tempo de travessia $\left(\frac{D_{i j}}{V_{\max _{i j}}}\right)$ em condições de trânsito livre.

$$
\text { peso }_{i j}=\frac{D_{i j}}{V_{\text {max }_{i j}}} \times n c_{i j}
$$

O processo de cálculo de uma nova rota consiste em dois estágios sendo esses: atualização dos pesos e cálculo do menor caminho. No primeiro, o veículo deve atualizar o custo das arestas do grafo referente as vias que ele possui informação utilizando a equação 2. Para as vias que o veículo não possui informação, é utilizado $N C_{i j}=1$, ou seja, considera-se que as vias possuem trânsito livre. No segundo estágio é executado um algoritmo de caminho mínimo (Dijkstra) para verificar a existência de rotas mais rápidas. É importante observar que, como a verificação de novas rotas é feita pelo veículo e cada veículo utiliza um conjunto diferente de informação sobre as vias (veículos em vias diferentes), não é necessário a execução de um algoritmo para encontrar $k$-rotas alternativas ou balanceamento de carga. O próprio paradigma distribuído da estruturação da solução proposta é capaz de realizar o balanceamento de rotas entre os veículos. 


\section{Resultado de Simulação}

A mobilidade dos veículos foi criada a partir da ferramenta SUMO $0.25^{1}$ e a comunicação entre veículos foi simulada utilizado o OMNET++ 5.1.12 em conjunto do framework de comunicação veicular Veins 4.3 [Sommer et al. 2011] que implementa o padrão IEEE 802.11p utilizado em redes veiculares. As simulações foram realizadas considerando um segmento do mapa da cidade de Los Angeles, EUA, sob uma região de $25 \mathrm{~km}^{2}{ }^{3}$ e cada veículo possui uma origem e destino escolhidos de maneira aleatória no mapa. Para verificar o comportamento das soluções, foram introduzidos diferentes quantidade de veículos no cenário: 1250, 2500, 3750, 5000 e 6250 veículos, sendo o primeiro aquele com poucos pontos de congestionamento e o último com muitos congestionamentos.

Para a análise de comunicação, foram utilizados uma largura de banda de $18 \mathrm{MBits} / \mathrm{s}$ para a cada MAC e potência de transmissão de $2.2 \mathrm{~mW}$, configurações que resultam em um alcance aproximado de 300 metros. Além disso, a Tabela 1 apresenta os valores avaliados e escolhidos para a solução proposta. Foram avaliados diferentes valores para NNC (Número de Níveis de Congestionamentos), T (intervalo de manutenção do conhecimento - Beacons) e $\Delta D E$ (variação da distância esperada). Foi definido o step $=1 s$ para o cálculo do Fator de Contenção (FC). Por fim, os resultados foram obtidos a partir de uma distribuição T-Student com média de 33 simulações e com o intervalo de confiança de 95\%. O intervalo de Beacons do PANDORA é de 2s, o mesmo utilizado pelos autores em [de Souza et al. 2017b].

A solução proposta (SGTD) foi comparada com as soluções da literatura RkSP, PANDORA, FOX e com o modelo sem a reorganização do tráfego (OVMT - Original Vehicle Mobility Traffic). Será apresentado uma análise referente a comunicação realizada pelos algoritmos distribuídos SGTD e PANDORA e uma análise referente as métricas de gerenciamento de tráfego com todos os algoritmos.

\begin{tabular}{lcr} 
Parâmetros & Avaliações & Melhor \\
\hline NNC & {$[5,10,15,20,30]$} & 10 \\
T $(\mathrm{s})$ & {$[5,10,20,30,40]$} & 10 \\
$\Delta D E(\%)$ & {$[50,60,70,80$ e 90$]$ de $D E$} & 80 \\
\hline
\end{tabular}

Tabela 1. Parâmetros do protocolo

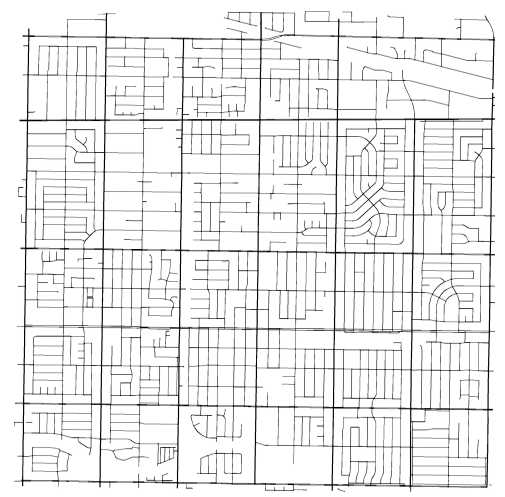

Figura 4. Mapa de Los Angeles

\subsection{Análise da Comunicação}

O objetivo dessa análise é avaliar o custo de comunicação das soluções distribuídas para o gerenciamento de tráfego. O desempenho foi avaliado considerando as seguintes métricas: Mensagens de conhecimento - número de mensagens transmitidas para a

\footnotetext{
${ }^{1}$ http://sumo.dlr.de/

${ }^{2}$ https://omnetpp.org/

${ }^{3}$ http://openstreetmap.org/
} 
criação de conhecimento, Transmissões totais - total de informações encaminhadas (incluindo as mensagens do tipo beacons utilizadas em ambas soluções), Colisões de Pacotes - pacotes que apresentam erros devido às colisões e, por fim, Número de Backoffs número de vezes em que os veículos entraram no modo Backoff na tentativa de acesso ao meio sem fio.

A Figura 5(a) descreve a relação entre o crescimento da quantidade de veículos e do número de mensagens de descoberta de conhecimento, excluindo as mensagens de manutenção do conhecimento (mensagens beacons). Ambas soluções apresentam comportamentos similares. Entretanto, considerando 3750 veículos o SGTD transmite 20\% menos mensagens em comparação com o PANDORA. A figura 5(b) apresenta a quantidade total de mensagens transmitidas por ambas soluções. Para realizar a manutenção do conhecimento, o PANDORA realiza a disseminação das mensagens do tipo Beacons dentro da região crítica (região onde é necessário manter conhecimento para realizar o gerenciamento de tráfego) a cada 2 segundos. Como o congestionamento no mapa avaliado pode acontecer em diversas áreas, várias regiões críticas são criadas pelo algoritmo, aumentando consideravelmente a quantidade de mensagens transmitidas pelo PANDORA. Diferentemente do PANDORA, o SGTD apenas necessita de informações das vias vizinhas para verificar a existência de rotas mais rápidas, onde os módulos Disseminação e Manutenção do Conhecimento conseguem reduzir consideravelmente a quantidade de mensagens para realizar o gerenciamento de tráfego. Em média, o PANDORA transmite $10^{2}$ vezes mais mensagens em comparação com o SGTD no cenário avaliado.

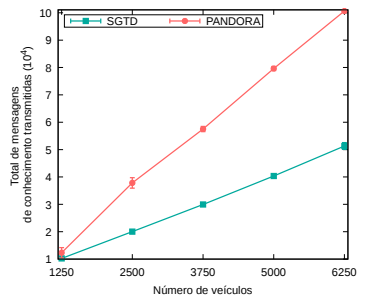

(a) Mensagens de conhecimento

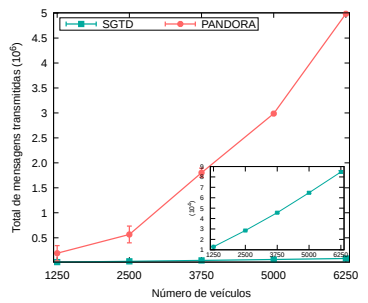

(b) Mensagens Totais

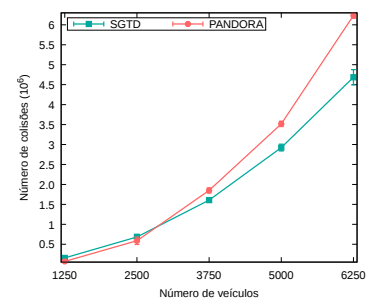

(c) Colisões de Pacotes

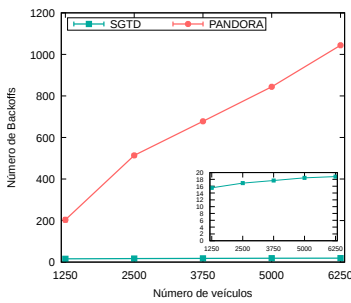

(d) Números de Backoff

Figura 5. Análise de Comunicação

A Figura 5(c) apresenta o número total de colisão de mensagens durante as simulações. Observa-se a correlação entre o aumento das transmissões de mensagens pelos algoritmos com o aumento da quantidade de colisões e isso é explicado pela sobrecarga no acesso ao meio sem fio. Pode-se perceber que o SGTD possui melhores resultados quando a rede possui muitos veículos ( $\geq 3750$ ). A quantidade total de colisão do SGTD é $16 \%$ e $26 \%$ menor em comparação com o PANDORA para 5000 e 6250 veículos respectivamente. A quantidade de backoffs média por veículo durante o seu percurso é apresentada na figura 5(d). Como a solução PANDORA realiza muitas transmissões, o acesso ao meio sem fio é constantemente disputado pelos veículos, onde a subcamada de acesso ao meio entra em modo backoff para prevenir transmissões simultâneas. Considerando 3750 veículos, cada veículo, em média, executa o backoff em torno de 650 vezes pelo PANDORA durante o seu percurso. Como a solução proposta apenas dissemina o conhecimento casa exista uma alteração nas condições de tráfego ou quando é necessário realizar a manutenção da informação sobre a via, um número menor de mensagens é ne- 
cessário para o seu funcionamento. Dessa forma, a disputa pelo meio sem fio na camada de enlace é menor. Em média, os veículos executando o SGTD entram em modo backoff 19 vezes durante o seu percurso considerando 6250 veículos.

\subsection{Análise do Deslocamento}

Nesta seção a solução proposta SGTD será avaliada em relação as soluções RkSP [Pan et al. 2017], PANDORA [de Souza et al. 2017b], FOX [Brennand et al. 2017] e OVMT considerando as seguintes métricas de avaliação de congestionamento: Tempo de Viagem - tempo médio necessário para os veículos concluírem o percurso, Distância média - distância média percorrida por cada veículo até o destino, Velocidade Média velocidade média dos veículos e Tempo de Congestionamento - tempo em que os veículos gastaram por causa de congestionamento.

A Figura 6(a) avalia o Tempo de Viagem e, quando a rede possui poucos pontos de congestionamento, todas as soluções possuem resultados similares (1250 veículos). A medida que a quantidade de veículos aumenta, o tempo de viagem aumenta por causa dos engarrafamentos e, por consequência, a diferença entre as soluções mostram-se evidentes. Quando o cenário possui 5000 veículos, o SGTD apresenta um tempo médio de viagem $5 \%, 23 \%, 28 \%$ e $30 \%$ inferior quando comparado às soluções RkSP, FOX, PANDORA e OVMT, respectivamente. Quando o cenário possui 6250 veículos, o desempenho do R $k$ SP e SGTD são similares. Entretanto, é importante ressaltar que o R $k$ SP é um serviço de gerenciamento de tráfego centralizado que possui uma visão global das condições de todas as vias do cenário, onde no SGTD os veículos apenas possuem informações sobre a sua vizinhança para o cálculo de novas rotas. Pode-se perceber também que a solução PANDORA não possui bons resultados em comparação com as demais soluções na presença de múltiplas regiões de congestionamento, possuindo resultados similares em comparação com as demais soluções apenas quando o cenário possui 1250 veículos.

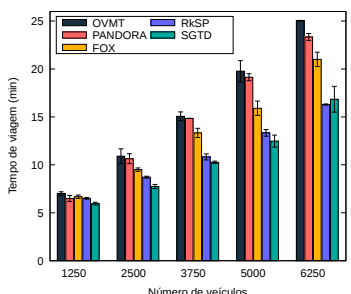

(a) Tempo de viagem

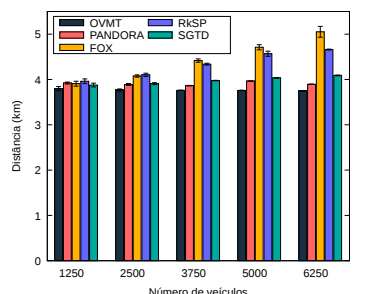

(b) Distância percorrida

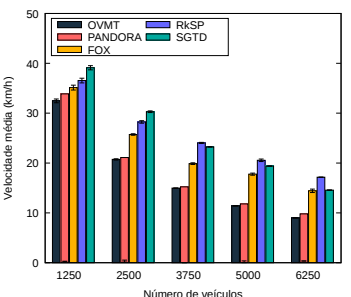

(c) Velocidade média

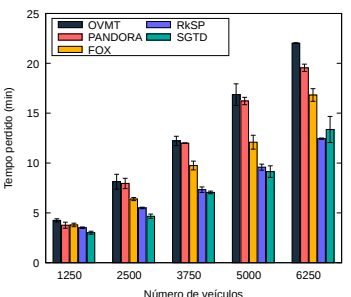

(d) Tempo perdido

Figura 6. Análise do deslocamento

Avaliando a distância média percorrida pelos veículos (figura 6(b)), o OVMT possui os melhores resultados, uma vez que os veículos trafegam nas menores rotas sem o controle de tráfego. Por outro lado, as soluções de gerenciamento de tráfego alteram os percursos dos veículos para reduzir o tempo de viagem e, assim, aumentam a distância percorrida. O FOX e R $k \mathrm{SP}$, que possuem uma visão maior sobre os congestionamentos, são capazes de fazer grandes mudanças no percurso dos veículos. Considerando 5000 veículos, a distância percorrida pelo FOX e RkSP é em torno de $13 \%$ superior em comparação com as demais soluções. Assim, o R $k$ SP e FOX conseguem diminuir a duração do trajeto, mas elevam consideravelmente a distância percorrida. As soluções 
distribuídas conseguem encontrar rotas alternativas com um pequeno aumento (em torno de 3\%) em relação ao percurso original (OVMT). É importante ressaltar que a abordagem proposta consegue reduzir o tempo de viagem com baixo impacto na distância percorrida, o que não é verificado pelo R $k$ SP.

A velocidade média dos veículos é apresentada na figura 6(c). O OVMT e PANDORA possuem velocidades médias semelhantes para diferentes quantidades de veículos no cenário. Para o cenário com poucos engarrafamentos, o SGTD é capaz de encontrar rotas alternativas com pequeno impacto na distância e sem engarrafamentos, proporcionando um aumento na velocidade média em comparação com as demais soluções. Entretanto, a medida que a quantidade de veículos aumenta, a solução R $k \mathrm{SP}$, utilizando o conhecimento global das condições do tráfego, consegue aumentar a velocidade média em $15 \%$ em comparação com o SGTD (considerando 6250 veículos). Avaliando as demais soluções para 6250 veículos, o SGTD possui resultados similares em comparação com o FOX e, em média, 50\% superiores em comparação ao PANDORA. O tempo perdido nos deslocamentos é avaliado na figura 6(d). Como o objetivo das abordagens para o gerenciamento de tráfego é encontrar rotas alternativas, é possível verificar que as soluções realmente conseguem atingir o objetivo, uma vez que o tempo perdido em engarrafamento é reduzido em todas as soluções. Considerando 3750 veículos, o SGTD reduz o tempo perdido em $2 \%, 25 \%, 40 \%$ e $43 \%$ em comparação com as soluções R $k$ SP, FOX, PANDORA E OVMT.

\section{Conclusões}

O presente trabalho apresenta o SGTD, um Sistema de Gerenciamento de Tráfego totalmente Distribuído para a redução de congestionamentos em centros urbanos. O SGTD utiliza a capacidade de comunicação de uma rede veicular para disseminar informações coletadas pelos veículos. Durante o seu percurso, o veículo verifica a contenção de deslocamento em sua via e, com base na contenção, é definido um nível de congestionamento para a via. A partir do nível de congestionamento das vias, os veículos, de maneira distribuída, verificam rotas alternativas com o objetivo de diminuir o tempo de viagem. Quando comparado com as abordagens da literatura, o SGTD possui resultados similares em comparação com a abordagem centralizada que possui a visão global da rede (R $k$ SP) e resultados superiores em comparação com as abordagens distribuída (PANDORA) e que utiliza computação em névoa (FOX). Como trabalhos futuros, pretende-se aprimorar a propagação do conhecimento de forma mais abrangente sem que, para isso, os ganhos alcançados pela solução sejam minimizados. Pretendemos também estudar outras funções na definição do fator de contenção (FC).

\section{Agradecimentos}

Os autores agradecem a Fundação de Amparo à Pesquisa do Estado de Minas Gerais (FAPEMIG) sob a concessão n..$^{\circ}$ APQ-03120-17 e ao Conselho Nacional de Desenvolvimento Científico e Tecnológico (CNPq) sob a concessão n. ${ }^{\circ}$ : 150545/2018-5. O presente trabalho foi realizado com apoio da Coordenação de Aperfeiçoamento de Pessoal de Nível Superior - Brasil (CAPES) - Código de Financiamento 001.

\section{Referências}

Ahmed, E. and Gharavi, H. (2018). Cooperative vehicular networking: A survey. IEEE Transactions on Intelligent Transportation Systems, 19(3):996-1014. 
Brennand, C., Boukerche, A., Meneguette, R., and Villas, L. (2017). A novel urban traffic management mechanism based on fog. IEEE Symposium on Computers and Communications (ISCC).

Cunha, F., Maia, G., Celes, C., Guidoni, D., de Souza, F., Ramos, H., and Villas, L. (2017). Sistemas de transporte inteligentes: Conceitos, aplicações e desafios. Minicursos do Simpósio Brasileiro de Redes de Computadores e Sistemas Distribuídos, 35(1/2017).

de Souza, A. M., Brennand, C. A., Yokoyama, R. S., Donato, E. A., Madeira, E. R., and Villas, L. A. (2017a). Traffic management systems: A classification, review, challenges, and future perspectives. International Journal of Distributed Sensor Networks, 13(4):1-14.

de Souza, A. M., da Fonseca, N. L. S., and Villas, L. A. (2017b). A fully-distributed advanced traffic management system based on opportunistic content sharing. 2017 IEEE International Conference on Communications (ICC), pages 1-6.

Dietzel, S., Petit, J., Kargl, F., and Scheuermann, B. (2014). In-network aggregation for vehicularad hocnetworks. IEEE Communications Surveys Tutorials, 16(4):1909-1932.

Lana, I., Ser, J. D., Velez, M., and Vlahogianni, E. I. (2018). Road traffic forecasting: Recent advances and new challenges. IEEE Intelligent Transportation Systems Magazine, 10(2):93-109.

ONU-HABITAT (2018). Programa das Nações Unidas para os Assentamentos Humanos. http://www. onuhabitat.org/.

Organization, W. H., UNAIDS, et al. (2006). Air quality guidelines: global update 2005. World Health Organization.

Pan, J., Popa, I. S., and Borcea, C. (2017). Divert: A distributed vehicular traffic rerouting system for congestion avoidance. IEEE Transactions on Mobile Computing, 16(1):58-72.

Pisa, I., Boquet, G., Vicario, J. L., Morell, A., and Serrano, J. (2018). Vaima: A v2v based intersection traffic management algorithm. In 2018 14th Annual Conference on Wireless On-demand Network Systems and Services (WONS), pages 125-128.

Scorecard, U. M. (2015). The texas a\&m transportation institute - urban mobility scorecard. https://mobility.tamu.edu/ums/report/.

Sommer, C., German, R., and Dressler, F. (2011). Bidirectionally Coupled Network and Road Traffic Simulation for Improved IVC Analysis. IEEE Transactions on Mobile Computing, 10(1):3-15.

Thai, J., Laurent-Brouty, N., and Bayen, A. M. (2016). Negative externalities of gpsenabled routing applications: A game theoretical approach. In 2016 IEEE 19th International Conference on Intelligent Transportation Systems (ITSC), pages 595-601.

Wu, C., Ji, Y., Liu, F., Ohzahata, S., and Kato, T. (2015). Toward practical and intelligent routing in vehicular ad hoc networks. IEEE Transactions on Vehicular Technology, 64(12):5503-5519. 